\title{
La búsqueda del círculo virtuoso de la Investigación, Desarrollo e Innovación
}

\author{
The search of the virtuous circle of Development, Research and Innovation
}

La búsqueda de un círculo virtuoso de la Investigación, Desarrollo e Innovación (I+D+i) desde un centro académico, implica establecer un modelo que permita identificar los componentes y la lógica de la cadena de valor.

El elemento central de la investigación lo constituyen los investigadores y los equipos de investigación, los mismos que actuando dentro de un ecosistema institucional favorable y apoyados en una infraestructura física, tecnológica y de gestión apropiada, desarrollan una cartera de proyectos.

Esta cartera de proyectos requiere para su concreción la identificación de fuentes de financiamiento nacionales y extranjeras, las mismas que se gestionan, con el apoyo institucional y llevan a la obtención de fondos de fuentes cooperantes, y la participación en Redes de I+D+i.

Estos fondos son aplicados al desarrollo de las actividades programadas en las investigaciones y el cumplimiento de los contratos efectuados y reciben para ello el soporte operativo, logístico, contable, administrativo, legal y económico necesario.

Durante el ciclo de vida de los proyectos de investigación científica y tecnológica, se obtienen productos y subproductos que dan cuenta de la generación, asimilación o adaptación de conocimiento científico y tecnológico, que se traducen en publicaciones, patentes y generación de paquetes para la transferencia tecnológica, algunos de ellos se concretan en emprendimientos e iniciativas empresariales desde la academia llamadas Spin off.

Estos productos requieren de un adecuado sistema de gestión de resultados que permita optimizar los beneficios para la sociedad, la institución y los investigadores, esto incluye aspectos de soporte legal, de propiedad intelectual, transferencia tecnológica, marketing y ventas; que haga sostenible la $\mathrm{I}+\mathrm{D}+\mathrm{i}$ en la Universidad.

Estos beneficios deben ser evaluados con un enfoque de Responsabilidad Social Universitaria y debiendo posibilitar la reinversión en el fortalecimiento de capacidades institucionales y mejorar el ecosistema institucional de $\mathrm{I}+\mathrm{D}+\mathrm{i}$, ampliando las posibilidades, de incluir nuevos investigadores a los equipos existentes, generar nuevos equipos de investigación y fortalecer las redes de investigación y la relación con las fuentes cooperantes.

En la medida que la cadena de valor muestre resultados, se mejorará el acceso a información y conocimiento; la identificación, captación y abordaje de los problemas y oportunidades de investigación científica y tecnológica; que luego se traduce en nuevos retos, para la institución, los investigadores, los equipos de investigación y las redes de investigación en las cuales participan y así sucesivamente. 
El proceso de Investigación Científica y Tecnológica, en la Universidad está influenciado por factores condicionantes externos que incluyen aspectos demográficos, económicos, socioculturales, políticos, legales, tecnológicos y ecológicos, que configuran un contexto de transformación y un escenario dinámico de globalización, competitividad, economía del conocimiento, innovaciones radicales e incrementales, que se traducen en nuevas oportunidades y demandas de los diferentes actores de la vida social y productiva, hacia el mundo académico.

El desarrollo de la función de la $\mathrm{I}+\mathrm{D}+\mathrm{i}$ en la Universidad, tiene por lo tanto que abordarse no solo desde el lado de la oferta de conocimientos, intereses y capacidades institucionales, sino que es necesario, considerar el enfoque de demanda del Estado, de la Empresa y de la Sociedad.

En la última década y a la par del crecimiento económico sostenido de nuestro país, se ha incrementado en términos reales la inversión en $\mathrm{I}+\mathrm{D}+\mathrm{i}$; la aplicación eficaz y eficiente de recursos por parte del Estado para desarrollar y fortalecer la cadena de producción científica y tecnológica configura una oportunidad para las universidades y para el sector productivo no sólo para promover la capacidad de innovación individual pero también para mejorar el nivel de interacción social propio de una sociedad innovadora.

Víctor Huanambal-Tiravanti ${ }^{1, a, b}$

Sección Odontología Restauradora y Biomateriales, Departamento Académico de Clínica Estomatológica, Facultad de Estomatología Roberto Beltrán, Universidad Peruana Cayetano Heredia. Lima, Perú.

Mg. Estomatología,

b Profesor. 